\title{
Processing, preservation and quality evaluation of sweetened Anardana
}

\author{
-G.P. ThORAT ${ }^{1 *}$, S.M. NAIKARE ${ }^{2}$, C. MANNURU ${ }^{2}$ AND W.A. KHAN ${ }^{1}$
}

${ }^{1}$ Department of Food Processing and Post-Harvest Technology, T.C. College, Baramati, PUNE (M.S.) INDIA

${ }^{2}$ Department of Food Science and Nutrition, S.N.D.T. College of Home Science, PUNE (M.S.) INDIA

*Author for Correspondence

Research chronicle : Received : 26.06.2015; Revised : 21.10.2015; Accepted : 23.11.2015

\section{SUMMARY :}

This study relates to the development of sweetened Anardana by using osmodehydration technology combining with coating of pomegranate arils and its quality evaluation during different storage conditions. Pomegranate fruits were subjected for blanching, then arils were separated carefully with minimum damage by hand peeling and further dipping arils in 40 per cent hypertonic sugar solution for overnight at room temperature $\left(25 \pm 2^{\circ} \mathrm{C}\right.$ for about $\left.14 \mathrm{hrs}\right)$ was conducted. Drained arils were partially dehydrated in a cabinet drier at $45 \pm 2^{\circ} \mathrm{C}$ for $16 \mathrm{hrs}$, subsequently coating with a mixture of glucose powder containing $\mathrm{CMC}(0.2 \%)$, sodium alginate $(0.1 \%)$ for binding and sodium citrate $(0.1 \%)$ for preservation followed by drying in cabinet dryer again. The step of coating and drying was repeated twice as moisture in arils was absorbing coating material and again appearing moist. Finally drying was done till desired level of moisture i.e. 10 per cent was attained. The experimental results showed that there was a significant loss in vitamin C content, slight decrease in moisture, ash, non-reducing sugars while there was increase in acidity, reducing sugars and total sugars. In case of refrigerated sample, the rate of change was significantly slower than the ambient sample. Microbial analysis showed that, there was increase in total plate count as well as yeast and mold count. Vacuum packed sample in multilayer bags i.e. standy pouches stored under refrigerated condition was found to be the best in terms of retaining chemical, microbial and sensory quality parameters over the storage period of 60 days.

KEY WORDS : Processing, Preservation, Quality evaluation, Sweetened Anardana

How to cite this paper : Thorat, G.P., Naikare, S.M., Mannuru, C. and Khan, W.A. (2015). Processing, preservation and quality evaluation of sweetened Anardana. Internat. J. Proc. \& Post Harvest Technol., 6 (2) $: 157-161$. 